\title{
Palladium- and Nickel-Catalyzed Aminations of Aryl Imidazolylsulfonates and Sulfamates
}

2011

Vol. 13, No. 7 1784-1786

\author{
Lutz Ackermann, ${ }^{*}$ René Sandmann, and Weifeng Song \\ Institut für Organische und Biomolekulare Chemie, Georg-August-Universität, \\ Tammannstrasse 2, 37077 Göttingen, Germany
}

Lutz.Ackermann@chemie.uni-goettingen.de

Received January 27, 2011

ABSTRACT

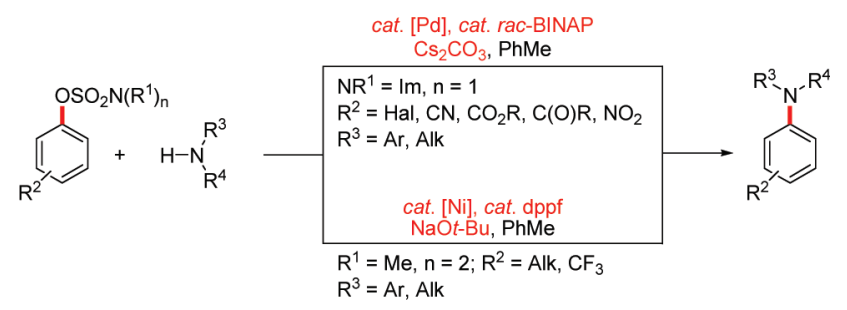

A nickel complex derived from dppf, along with $\mathrm{NaOt-Bu}$ as the base, allowed for challenging aminations of aryl sulfamates. An improved functional group tolerance is observed in novel palladium-catalyzed aminations of imidazolylsulfonates with rac-BINAP as the ligand.

Transition-metal-catalyzed arylations of amines with aryl halides are among the most important methods for the selective formation of $\mathrm{C}-\mathrm{N}$ bonds. ${ }^{1}$ Particularly, the use of phenol-derived electrophiles in catalyzed arylations is highly attractive, ${ }^{2}$ since they are readily accessible and can be easily implemented as directing groups in site-selective arene functionalization strategies. ${ }^{2,3}$ However, the inherently high $\mathrm{C}-\mathrm{O}$ bond strength in phenols calls for an activation of these precursors, which was largely achieved through the use of rather expensive fluorine-containing reagents. ${ }^{4}$ On the contrary, recent progress in the use of phenol-derived electrophiles in catalytic arylations was represented by the use of

(1) (a) Surry, D. S.; Buchwald, S. L. Chem. Sci. 2011, 2, 27-50. (b) Klinkenberg, J. L.; Hartwig, J. F. Angew. Chem., Int. Ed. 2011, 50, 8695. (c) Monnier, F.; Taillefer, M. Angew. Chem., Int. Ed. 2009, 48, 6954 6971. (d) Krüger, K.; Tillack, A.; Beller, M. Adv. Synth. Catal. 2008, 350, 2153-2167. (e) Ackermann, L. Synlett 2007, 507-526. (f) Schlummer, B.; Scholz, U. Adv. Synth. Catal. 2004, 346, 1599-1626.

(2) (a) Rosen, B. M.; Quasdorf, K. W.; Wilson, D. A.; Zhang, N.; Resmerita, A.-M.; Garg, N. K.; Percec, V. Chem. Rev. 2011, DOI: 10.1021/cr100259t. (b) Li, B.-J.; Yu, D.-G.; Sun, C.-L.; Shi, Z.-J. Chem.-Eur. J. 2011, 17, 1728-1759. (c) Yu, D.-G.; Li, B.-J.; Shi, Z.-J. Acc. Chem. Res. 2010, 43, 1486-1495. (d) Littke, A. In Modern Arylation Methods; Ackermann, L., Ed.; Wiley-VCH: Weinheim, 2009; pp 25-67.

(3) Hartung, C. G.; Snieckus, V. In Modern Arene Chemistry; Astruc, D., Ed.; Wiley-VCH: Weinheim, 2002; pp 330-367.

(4) Select reviews: (a) Högermeier, J.; Reissig, H.-U. Adv. Synth. Catal. 2009, 351, 2747-2763. (b) Zeni, G.; Larock, R. C. Chem. Rev. 2006, 106, 4644-4680. (c) Stang, P. J.; Hanack, M.; Subramanian, L. R. Synthesis 1982, 85-126. imidazolylsulfonates and sulfamates, because of their airand moisture-stable nature, their attractive handling properties, and their low costs. ${ }^{5,6}$ While these user-friendly electrophiles were recently employed for efficient $\mathrm{C}-\mathrm{C}$ bond formations, their use in transition-metal-catalyzed aminations

(5) For representative examples of catalyzed arylations with tosylates or mesylates, see: $\mathrm{C}-\mathrm{N}$ bond formation: (a) Mantel, M. L. H.; Lindhardt, A. T.; Lupp, D.; Skrydstrup, T. Chem.-Eur. J. 2010, 16, $5437-$ 5442. (b) Lundgren, R. J.; Stradiotto, M. Angew. Chem., Int. Ed. 2010, 49, 8686-8690. (c) So, C. M.; Zhou, Z.; Lau, C. P.; Kwong, F. Y. Angew. Chem., Int. Ed. 2008, 47, 6402-6406. (d) Nguyen, H. N.; Huang, X.; Buchwald, S. L. J. Am. Chem. Soc. 2003, 125, 11818-11819. (e) Roy, A. H.; Hartwig, J. F. J. Am. Chem. Soc. 2003, 125, 8704-8705. (f) Huang, X.; Anderson, K. W.; Zim, D.; Jiang, L.; Klapars, A.; Buchwald, S. L. J. Am. Chem. Soc. 2003, 125, 6653-6655. (g) Bolm, C.; Hildebrand, J. P.; Rudolph, J. Synthesis 2000, 911-913. (h) Hamann, B. C.; Hartwig, J. F. J. Am. Chem. Soc. 1998, 120, 7369-7370 and references cited herein. See also: (i) Ackermann, L.; Fenner, S. Chem. Commun. 2011, 47, 430-432. (j) Yeung, P. Y.; So, C. M.; Lau, C. P.; Kwong, F. Y. Angew. Chem., Int. Ed. 2010, 49, 8918-8922. (k) Wilson, D. A.; Wilson, C. J.; Moldoveanu, C.; Resmerita, A.-M.; Corcoran, P.; Hoang, L. M.; Rosen, B. M.; Percec, V. J. Am. Chem. Soc. 2010, 132, 1800-1801. (1) Chow, W. K.; So, C. M.; Lau, C. P.; Kwong, F. Y. J. Org. Chem. 2010, 75, 5109-5112. (m) Bhayana, B.; Fors, B. P.; Buchwald, S. L. Org. Lett. 2009, 11, 3954 3957. (n) Ackermann, L.; Althammer, A.; Fenner, S. Angew. Chem., Int . Ed. 2009, 48, 201-204. (o) So, C. M.; Lau, C. P.; Kwong, F. Y. Angew. Chem., Int. Ed. 2008, 47, 8059-8063. (p) Zhang, L.; Wu, J. Adv. Synth. Catal. 2008, 350, 2409-2413. (q) Zhang, L.; Wu, J. J. Am. Chem. Soc. 2008, 130, 12250-12251. (r) Hansen, A. L.; Ebran, J.-P.; Ahlquist, M.; Norrby, P.-O.; Skrydstrup, T. Angew. Chem., Int. Ed. 2006, 45, 33493353. (s) Ackermann, L.; Althammer, A. Org. Lett. 2006, 8, 3457-3460. (t) Zhou, J.; Fu, G. C. J. Am. Chem. Soc. 2003, 125, 12527-12530 and references cited therein. 
has unfortunately thus far proven elusive. Within our research program on the development of efficient amination reactions for modular heteroarene syntheses, ${ }^{7}$ we thus explored various transition metal complexes for catalyzed aminations with aryl sulfamates and imidazolylsulfonates, on which we report herein.

At the outset, we tested various ligands and bases in the nickel-catalyzed amination of sulfamate 1a (Table 1). While representative monodentate phosphine ligands provided only unsatisfactory results (entries $1-5$ ), nickel complexes derived from $\mathrm{N}$-heterocyclic carbene (NHC) precursors (entries 6 and 7) or bidentate phosphine ligands (entries 8-14) displayed improved catalytic activities. Notably, the best results were accomplished with dppf as the ligand, along with $\mathrm{NaO} t$ - $\mathrm{Bu}$ as the base (entries 12-14).

Table 1. Optimization of Nickel-Catalyzed Amination ${ }^{a}$

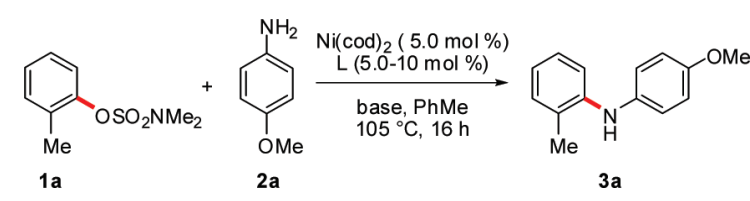

\begin{tabular}{|c|c|c|c|}
\hline entry & $\mathrm{L}(\mathrm{mol} \%)$ & base & yield $(\%)$ \\
\hline 1 & - & $\mathrm{NaO} t-\mathrm{Bu}$ & - \\
\hline 2 & $\mathrm{PPh}_{3}(10)$ & $\mathrm{NaO} t-\mathrm{Bu}$ & - \\
\hline 3 & $\mathrm{PCy}_{3}(10)$ & $\mathrm{NaO} t-\mathrm{Bu}$ & - \\
\hline 4 & S-Phos (10) & $\mathrm{NaO} t-\mathrm{Bu}$ & - \\
\hline 5 & X-Phos (10) & $\mathrm{NaO} t-\mathrm{Bu}$ & - \\
\hline 6 & $\mathrm{IPrHCl}(10)$ & $\mathrm{NaO} t-\mathrm{Bu}$ & 85 \\
\hline 7 & SIPrHCl (10) & $\mathrm{NaO} t-\mathrm{Bu}$ & 82 \\
\hline 8 & rac-BINAP (5.0) & $\mathrm{NaO} t-\mathrm{Bu}$ & 54 \\
\hline 9 & 1,10-phenanthroline (5.0) & $\mathrm{NaO} t-\mathrm{Bu}$ & 8 \\
\hline 10 & dppe (5.0) & $\mathrm{NaO} t-\mathrm{Bu}$ & 52 \\
\hline 11 & $\operatorname{dppp}(5.0)$ & $\mathrm{NaO} t-\mathrm{Bu}$ & - \\
\hline 12 & $\operatorname{dppf}(5.0)$ & $\mathrm{KO} t-\mathrm{Bu}$ & 65 \\
\hline 13 & $\operatorname{dppf}(5.0)$ & $\mathrm{Cs}_{2} \mathrm{CO}_{3}$ & - \\
\hline 14 & $\operatorname{dppf}(5.0)$ & $\mathrm{NaO} t-\mathrm{Bu}$ & 95 \\
\hline
\end{tabular}

${ }^{a}$ Reaction conditions: 1a $(0.50 \mathrm{mmol}), \mathbf{2 a}(0.75 \mathrm{mmol}), \mathrm{Ni}(\operatorname{cod})_{2}(5.0$ $\mathrm{mol} \%), \mathrm{L}(5.0-10 \mathrm{~mol} \%)$, base $(0.75 \mathrm{mmol}), \mathrm{PhMe}(2.0 \mathrm{~mL}), 105^{\circ} \mathrm{C}, 16 \mathrm{~h}$; S-Phos = 2-dicyclohexylphosphino-2',6'-dimethoxybiphenyl; X-Phos = 2-dicyclohexylphosphino-2 $2^{\prime} 4^{\prime}, 6^{\prime}$-tri-iso-propylbiphenyl; (S)IPrH $=N, N^{\prime}$ bis-(2,6-di-iso-propylphenyl)imidazol(in)ium.

With an optimized catalytic system in hand, we explored its scope in the nickel-catalyzed amination of differently substituted sulfamates 1 (Scheme 1). Importantly, aniline derivatives bearing electron-withdrawing as well as electron-donating substituents efficiently provided the desired products $\mathbf{3 b}-\mathbf{3 i}$, even when being sterically hindered. Moreover, challenging $n$-alkyl amines were converted with comparable catalytic efficacy.

For alkyl-substituted secondary amines, a nickel complex generated in situ from an NHC precursor constituted a viable alternative (Scheme 2).

While the optimized nickel complexes displayed promising catalytic activities, they relied on air-sensitive $\mathrm{Ni}(\mathrm{cod})_{2}$,
Scheme 1. Nickel-Catalyzed Amination of Sulfamtes 1

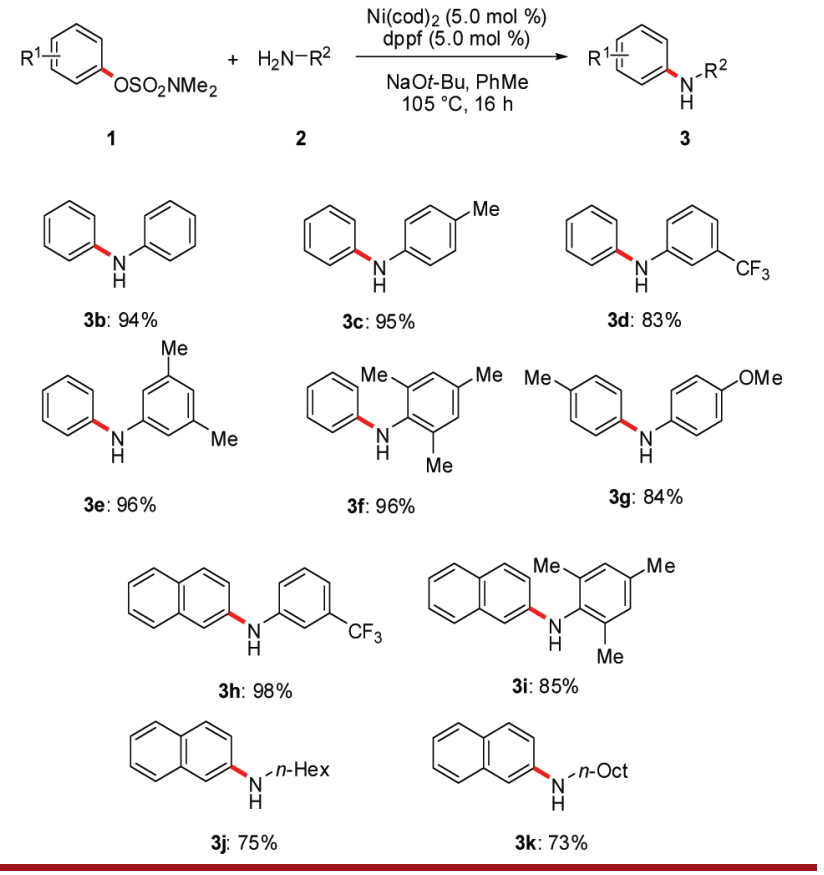

Scheme 2. Nickel-Catalyzed Arylation of Secondary Amine 2b

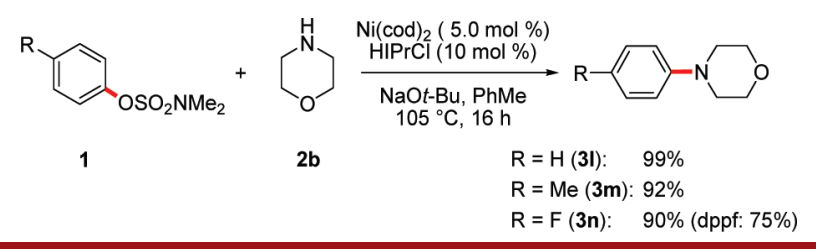

and their functional group tolerance was significantly restricted by the required strong base $\mathrm{NaO} t$ - $\mathrm{Bu}$. Therefore, we subsequently tested various palladium catalysts for the amination of imidazolylsulfonate $\mathbf{4 a}$ using milder bases (Table 2). Unfortunately, various NHC palladium complexes gave only unsatisfactory yields (entries $1-4)$. However, among a variety of phosphine ligands (entries 5-16),

(6) (a) Macklin, T. K.; Snieckus, V. Org. Lett. 2005, 7, 2519-2522. (b) When, P. M.; Du Bois, J. Org. Lett. 2005, 7, 4685-4688. (c) AlbanezeWalker, J.; Raju, R.; Vance, J. A.; Goodman, A. J.; Reeder, M. R.; Liao, J.; Maust, M. T.; Irish, P. A.; Espino, P.; Andrews, D. R. Org. Lett. 2009, 11, 1463-1466. (d) Goegsig, T. M.; Lindhardt, A. T.; Skrydstrup, T. Org. Lett. 2009, 11, 4886-4888. (e) Ackermann, L.; Barfüsser, S.; Pospech, J. Org. Lett. 2010, 12, 724-726. (f) Luo, Y.; Wu, J. Organometallics 2009, 28, 6823-6826. (g) Shirbin, S. J.; Boughton, B. A.; Zammit, S. C.; Zanatta, S. D.; Marcuccio, S. M.; Hutton, C. A.; Williams, S. J. Tetrahedron Lett. 2010, 51, 2971-2974. (h) Quasdorf, K. W.; Riener, M.; Petrova, K. V.; Garg, N. K. J. Am. Chem. Soc. 2009, 131, 1774817749 and references cited therein. (i) For nickel-catalyzed aminations of aryl pivalates, see: Shimasaki, T.; Tobisu, M.; Chatani, N. Angew. Chem., Int. Ed. 2010, 49, 2929-2932. (j) After submission of our manuscript, a nickel-catalyzed amination of aryl sulfamates was reported by Garg and coworkers: Ramgren, S. D.; Silberstein, A. L.; Yang, Y.; Garg, N. K. Angew. Chem., Int. Ed. 2011, 50, DOI: 10.1002/anie.201007325. 
Table 2. Optimization of Palladium-Catalyzed Amination ${ }^{a}$

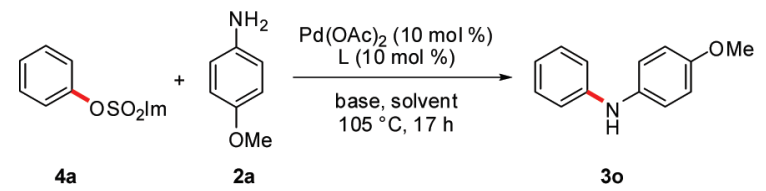

$4 a$

$2 a$

\begin{tabular}{lllll}
\hline entry & \multicolumn{1}{c}{$\mathrm{L}$} & \multicolumn{1}{c}{ base } & \multicolumn{1}{c}{ solvent } & yield (\%) \\
\hline 1 & - & $\mathrm{Cs}_{2} \mathrm{CO}_{3}$ & $\mathrm{PhMe}$ & - \\
2 & sHIPrCl & $\mathrm{Cs}_{2} \mathrm{CO}_{3}$ & $\mathrm{PhMe}$ & $5^{b}$ \\
3 & $\mathrm{HIPrCl}$ & $\mathrm{Cs}_{2} \mathrm{CO}_{3}$ & $\mathrm{PhMe}$ & $9^{b}$ \\
4 & $\mathrm{HIMesCl}$ & $\mathrm{Cs}_{2} \mathrm{CO}_{3}$ & $\mathrm{PhMe}$ & - \\
5 & $\mathrm{PPh}$ & $\mathrm{Cs}_{2} \mathrm{CO}_{3}$ & $\mathrm{PhMe}$ & - \\
6 & John-Phos & $\mathrm{Cs}_{2} \mathrm{CO}_{3}$ & $\mathrm{PhMe}$ & 83 \\
7 & X-Phos & $\mathrm{Cs}_{2} \mathrm{CO}_{3}$ & $\mathrm{PhMe}$ & 90 \\
8 & X-Phos & $\mathrm{Cs}_{2} \mathrm{CO}_{3}$ & $\mathrm{PhMe}$ & $79^{c}$ \\
9 & dppe & $\mathrm{Cs}_{2} \mathrm{CO}_{3}$ & $\mathrm{PhMe}$ & - \\
10 & dppf & $\mathrm{Cs}_{2} \mathrm{CO}_{3}$ & $\mathrm{PhMe}$ & $8^{b}$ \\
11 & rac-BINAP & $\mathrm{Cs}_{2} \mathrm{CO}_{3}$ & $\mathrm{THF}$ & 64 \\
12 & rac-BINAP & $\mathrm{Cs}_{2} \mathrm{CO}_{3}$ & $1,4-d i o x a n e$ & 80 \\
13 & rac-BINAP & $\mathrm{K}_{3} \mathrm{PO}_{4}$ & $\mathrm{PhMe}$ & 58 \\
14 & rac-BINAP & $\mathrm{K}_{2} \mathrm{CO}_{3}$ & PhMe & $29^{b}$ \\
15 & rac-BINAP & $\mathrm{Na}_{2} \mathrm{CO}_{3}$ & PhMe & - \\
16 & rac-BINAP & $\mathrm{Cs}_{2} \mathrm{CO}_{3}$ & PhMe & 86
\end{tabular}

${ }^{a}$ Reaction conditions: 4a $(0.50 \mathrm{mmol}), 2 \mathrm{a}(0.60-0.75 \mathrm{mmol})$, $\mathrm{Pd}(\mathrm{OAc})_{2}(10 \mathrm{~mol} \%), \mathrm{L}(10 \mathrm{~mol} \%)$, base $(0.65-1.25 \mathrm{mmol})$, solvent $(2.0 \mathrm{~mL}), 105^{\circ} \mathrm{C}, 17 \mathrm{~h}$; John-Phos $=2$-di(tert-butyl)phosphinobiphenyl. ${ }^{b} \mathrm{GC}$ conversion. ${ }^{c} \mathrm{Pd}(\mathrm{OAc})_{2}(2.0 \mathrm{~mol} \mathrm{\%}), \mathrm{L}(4.0 \mathrm{~mol} \%) .{ }^{d} 65^{\circ} \mathrm{C}$.

particularly X-Phos ${ }^{8}$ and $r a c$-BINAP turned out to be superior (entries 11-16).

The optimized reaction conditions were thereafter employed for evaluating the scope of palladium-catalyzed aminations of imidazolylsulfonates 4 (Scheme 3). Notably, differently substituted aniline derivatives were successfully converted, as was an alkyl-substituted amine. Contrary to reactions with nickel complexes (vide supra), the palladiumcatalyzed amination proved tolerant of valuable electrophilic functional groups, such as fluoro-, nitro-, or cyano-substituents, as well as ester or ketone functionalities. An additional valuable asset of the catalytic system is inter alia represented by its chemoselectivity in the conversion of chloro-substituted aryl imidazolylsulfonates $\mathbf{4}$, which is complementary to the one observed in palladium-catalyzed aminations with aryl tosylates $^{5 \mathrm{~d}}$ or mesylates ${ }^{5 \mathrm{c}}$ as electrophiles.

When using electron-rich aryl imidazolylsulfonates, a palladium complex of phosphine ligand X-Phos delivered high yields as well (Scheme 4).

(7) For representative examples, see: (a) Ackermann, L.; Song, W.; Sandmann, R. J. Organomet. Chem. 2011, 696, 195-201. (b) Ackermann, L.; Barfüsser, S.; Potukuchi, H. K. Adv. Synth. Catal. 2009, 351, 1064 1072. (c) Ackermann, L.; Sandmann, R.; Kaspar, L. T. Org. Lett. 2009, 11, 2031-2034. (d) Ackermann, L.; Sandmann, R.; Kondrashov, M. V. Synlett 2009, 1219-1222. (e) Ackermann, L.; Sandmann, R.; Villar, A.; Kaspar, L. T. Tetrahedron 2008, 64, 769-777. (f) Ackermann, L.; Spatz, J. H.; Gschrei, C. J.; Born, R.; Althammer, A. Angew. Chem., Int. Ed. 2006, 45, 7627-7630. (g) Ackermann, L.; Born, R. Angew. Chem., Int. Ed. 2005, 44, 2444-2447 and references cited therein.
Scheme 3. Scope of Palladium-Catalyzed Aminations of Imidazolylsulfonates 4
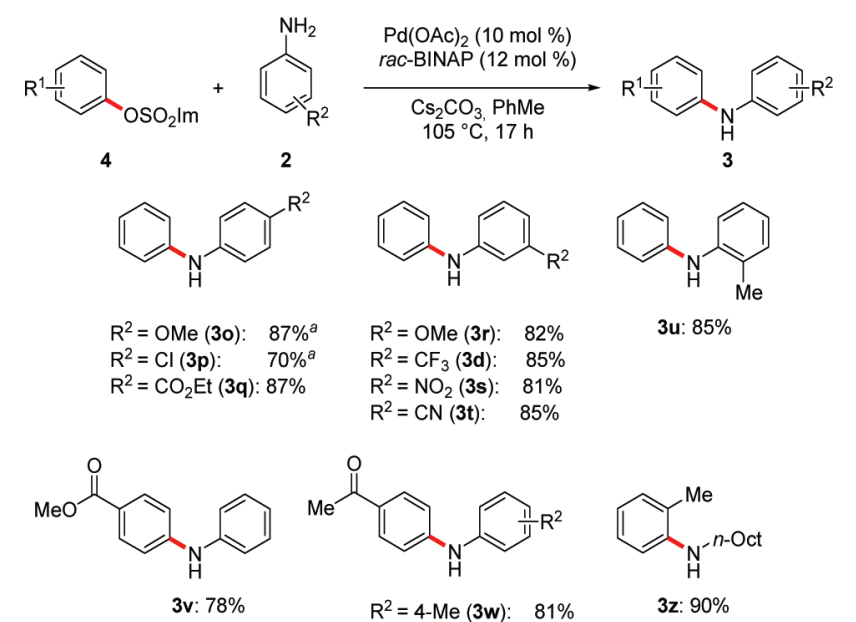

$\mathrm{R}^{2}=4-\mathrm{Me}(3 \mathrm{w}): \quad 81 \%$ $\mathrm{R}^{2}=3-\mathrm{MeO}(\mathbf{3 x}): 76 \%$

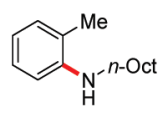

$\mathrm{R}^{2}=2-\mathrm{F}(3 \mathrm{y}): \quad 86 \%$

3z: $90 \%$

${ }^{a}[\mathrm{Pd}](5.0 \mathrm{~mol} \%)$.

Scheme 4. Palladium-Catalyzed Amination of Electron-Rich Imidazolylsulfonate $\mathbf{4 b}$

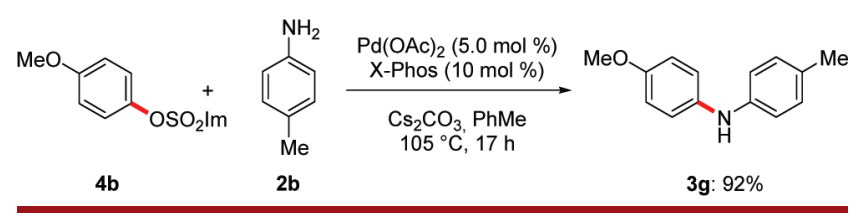

In summary, we have disclosed unprecedented general metal-catalyzed aminations of aryl sulfamates and imidazolylsulfonates. Hence, a nickel catalyst derived from ligand dppf allowed for arylations with challenging sulfamates as electrophiles, provided that $\mathrm{NaO} t$-Bu was used as the base. On the contrary, palladium complexes of racBINAP set the stage for widely applicable aminations of aryl imidazolylsulfonates, a notable feature of which is constituted by their excellent functional group tolerance. Further studies on applications of these electrophiles in catalytic arylations are currently ongoing in our laboratories and will be reported in due course.

Acknowledgment. Support by the DFG and the Chinese Scholarship Council (fellowship to W.S.) is gratefully acknowledged.

Supporting Information Available. Experimental procedures, characterization data, and ${ }^{1} \mathrm{H}$ and ${ }^{13} \mathrm{C}$ NMR spectra for new compounds. This material is available free of charge via the Internet at http://pubs.acs.org.

(8) Surry, D. S.; Buchwald, S. L. Angew. Chem., Int. Ed. 2008, 47, 6338-6361. 